\title{
Conservative Management of Pulmonary Artery Dissection
}

\author{
S. Togo', M. A. Ouattara1, I. Sankaré1, S. Yéna1, N. Diani², A. M. Camara3 \\ ${ }^{1}$ Thoracic Surgery Department, Hospital of Mali, Bamako, Mali \\ ${ }^{2}$ Department of Reanimation (Intensive Care Unit), Hospital of Mali, Bamako, Mali \\ ${ }^{3}$ Department of Radiology, Hospital of Mali, Bamako, Mali \\ Email: drseydoutg@yahoo.fr
}

Received 20 June 2015; accepted 18 July 2015; published 21 July 2015

Copyright (C) 2015 by authors and Scientific Research Publishing Inc.

This work is licensed under the Creative Commons Attribution International License (CC BY).

http://creativecommons.org/licenses/by/4.0/

c) (7) Open Access

\begin{abstract}
The pulmonary artery dissection (PAD) is a rare and life-threatening event, usually secondary to a chronic pulmonary artery hypertension. We report the case of pulmonary artery dissection undergoing medical treatment by conservative management, discovered by performing a computed tomography (CT), and describe the clinical and therapeutic characteristics with the literature review.
\end{abstract}

\section{Keywords}

Pulmonary Artery, Dissection, Computed Tomography, Conservative Management

\section{Introduction}

The PAD is a rare and life threatening event and not well known disease [1]. Usually it occurs as a complication of chronic pulmonary artery hypertension leading to pulmonary artery aneurysm. However, other causes have been involved in the etiology of this disease [2]. Although arterial dissection commonly occurs in the aorta, it is very unusual in the pulmonary arteries [1] [2]. The improvements in imaging techniques have resulted in earlier diagnoses. A definitive approach of PAD treatment has not yet been established [3]. We report a case of PAD undergoing medical treatment by conservative management and describe the clinical and therapeutic characteristics with the literature review.

\section{Case Presentation}

An 88-year-old woman was referred to emergencies and hospitalized in intensive care unit on 29 September 
2014, after a sudden loss of conscious associated with a transient sharp chest pain, worsening dyspnea and cough since one week. Chest radiography and a cardiac ultrasound scan performed in a private hospital concluded a massive pulmonary embolism. She was misdiagnosed and underwent initial treatment for pure embolism. Her past medical history was positive for high blood pressure associate with chronic pulmonary hypertension treated medically and traditionally for 23 years. There was no risk factor of diabetes. Her husband is a smoker for 30 years. Since 5 years, she had a walk perimeter less than $50 \mathrm{~m}$. The general condition was altered with a classification of New York Heart Association quoted to 3. The temperature was $37.2^{\circ} \mathrm{C}$, heart rate was 160 beats/min, blood pressure was 190/110 $\mathrm{mm} \mathrm{Hg}$, respiration frequency was $32 \mathrm{cycles} / \mathrm{min}$. The oxygen saturation was $85 \%$ on room air. A systolic heart murmur at the mitral valve auscultation was listening. The breath sounds were diminished in both lung fields. The patient was conscious at admission but his condition worsened quickly with a low blood pressure ( $\mathrm{BP}=40 / 20 \mathrm{mmHg}$ ) followed by coma which justified the urgent intubation in intensive care unit with the use of inotropic electric syringe (dobutamine and noradrenaline) and a high concentration oxygen mask. The initial biological assessment D-dimer is normal (320 micrograms/L.) The antistreptolysin test, RPR syphilis serology, Intra dermo-reaction and sputum for tuberculosis check up were negative. Cerebral and thoracic CT has eliminated brain damage and pulmonary embolism, respectively.

However contrast CT scan showed that the pulmonary artery was enlarged at the level of the pulmonary arterial tree, reaching a maximum diameter of $48 \mathrm{~mm}$. We noted a pulmonary artery dissection at the level of the proximal tree with a posterior false lumen and a reduced diameter of a true lumen (Figure 1). The right pulmonary artery (RPA) and left pulmonary artery (LPA) branches measured 5 and $4 \mathrm{~cm}$, respectively and were also affected by the dissection associated with a minimal bilateral pleural effusion (Figure 2). A dissection flap was noted in the posterior aspect of the pulmonary artery above the valve. There was no pericardial effusion or hematoma, or any other sign of rupture. There is no thrombus formation in the PA. The RPA was more dissected in distally compare to the LPA (Figure 3). Echocardiography performed in ICU found a dilated hypertrophic right ventricle with good left ventricular function $(\mathrm{EF}=60 \%)$. There was a blood circulation in the pulmonary artery with an estimated pulmonary systolic pressure of $75 \mathrm{mmHg}$ also associated with tricuspid valve insufficiency. Cardiomegaly and a big liver were also found. In transesophageal echocardiography, the pulmonary artery and the branches were dilated but the blood circulation distally in the RPA and LPA was difficult to analyze. The treatment conducted was the use of antihypertensive vasodilator by intravenous injection, an anticoagulant (low molecular weight heparin) to preventive dose, prostacyclin and diuretic for 14 days. An antibiotic amoxicillin-based + clavulanic acid has been used for 7 days. The outcome was favorable; the patient was extubated after 10 days and received a new cardiological examination. Heart sounds were regular. Oxygen therapy was still

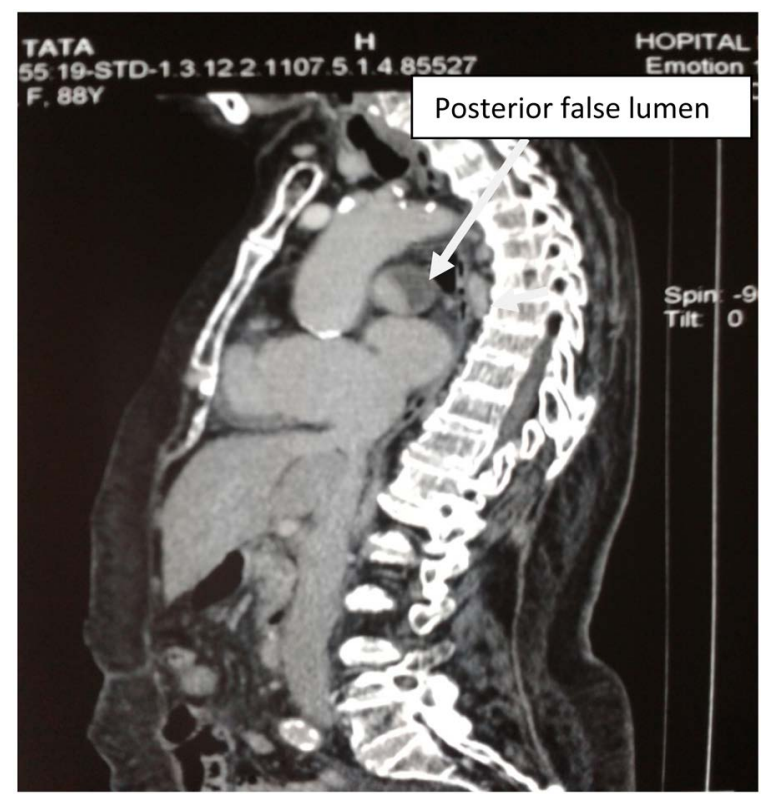

Figure 1. Dissection of the proximal pulmonary artery trunk with a posterior false lumen. 


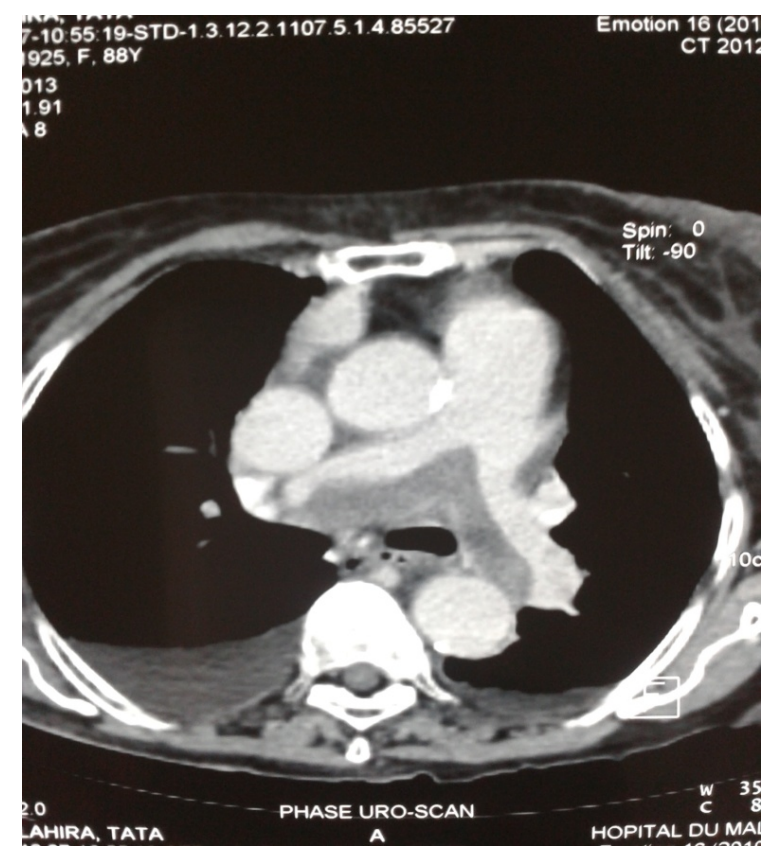

Figure 2. Right and left pulmonary artery dissection associated with bilateral pleural effusion.

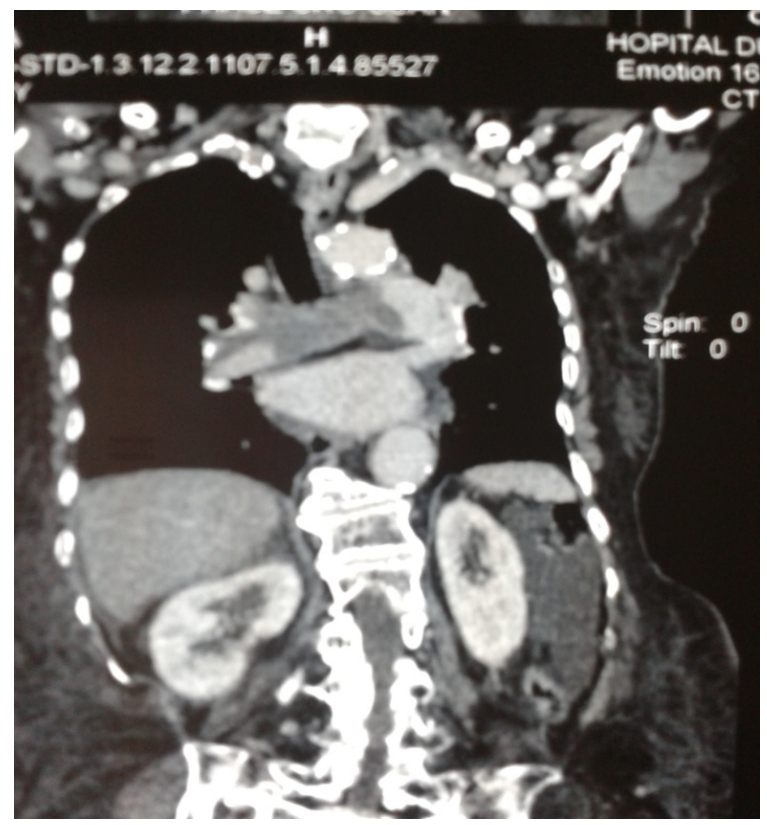

Figure 3. Pulmonary artery dissected distally but more pronounced on the right artery trunk.

given. The electrocardiogram showed left ventricular hypertrophy. Echocardiography control found the pulmonary artery aneurysm extended to the origin of the left and right pulmonary artery. The diameter measured 41 $\mathrm{mm}$ (previously $48 \mathrm{~mm}$ in admission). Left ventricular function still normal $(\mathrm{EF}=60 \%)$ The pulmonary hypertension (55 mmhg) remains and there was no valvular abnormality. In the absence of cardiovascular surgery medical equipments in the country to achieve the surgery, and the lack of financial means to continue a treatment abroad, the patient was discharged with medical oral treatment including wafarin, prostacyclin, colosar (antihypertensive), diuretic and oxygen therapy by oxygen extractor device. A three and six month's cardiologi- 
cal control confirms the stability of the blood pressure. Recent CT control performed revealed the PAD (Figure 4). To date, the patient is still alive. She was hemodynamically stable and complained only of nonspecific respiratory symptoms.

\section{Discussion}

Based on the clinical history, the dissection of the pulmonary artery may have been precipitated by an acquired pulmonary infection and worsen into a hypoxia thereby increasing pulmonary artery pressure. Hemoptysis up to cataclysmic hemorrhage is often described in the symptoms and the occurrence is witness to the instability of the lesion [1]. Sometimes pulmonary artery hypertension, right heart failure or heart murmur were the current symptoms. The clinical diagnosis of PAD can be difficult because of nonspecific signs and sometimes only confusing cough, dyspnea, chest pain or cyanosis was found [4]. The dyspnea and chest pain were the mains symptoms in our patient. Aneurysms are often associated with infection (typically tuberculosis and syphilis), trauma, damage of the vessel wall (the media necrosis or atherosclerosis), hereditary metabolic disorders of the elastic tissue and pulmonary artery hypertension. Marfan syndrome, Behcet's diseases are also involved [2]. The infectious origin is usually associated with a particular field such as patent ductus artery, atrial or inter ventricular communication, tetralogy of Fallot and pulmonary stenosis. These congenital heart defects support strong intra pulmonary pressures that will create the chronic pulmonary hypertension [4]. The majority of pulmonary artery dissection occurs in the presence of an internal degeneration associated with fragmentation of elastic fibers and a general expansion of the pulmonary arterial tree caused by chronic pulmonary hypertension [5]. We found the pulmonary hypertension as the contributing factor in our patient who had high blood pressure poorly treated with a compatible cardiac hypertrophy. The pulmonary artery trunk is the main site of dissection in $80 \%$ of cases, usually without the participation of the branches; the other cases are isolated in the right or left branch or intrapulmonary branches [6]. In a few cases, the pulmonary artery dissection can be located in the site of aneurysm formation [7]. In PAD the false lumen in the pulmonary artery dissection tends to break rather than extending distally [6] [8]. In the last two centuries, 64 other cases of pulmonary artery dissection have been described only eight of them were diagnosed from living patients [8]. Pulmonary arteriography, CT scan, and magnetic resonance imaging (MRI) can detect an intimal flap and a false lumen within the pulmonary arterial tree, and are able to evaluate the extent of the lesion [9]. These methodologies can provide additional information related to the dissection and the presence of associated abnormal findings such as pericardial tamponade [10]. CT scan also measures the diameter and the aneurysm extent and can exclude the presence of pulmonary embolism [11]. The

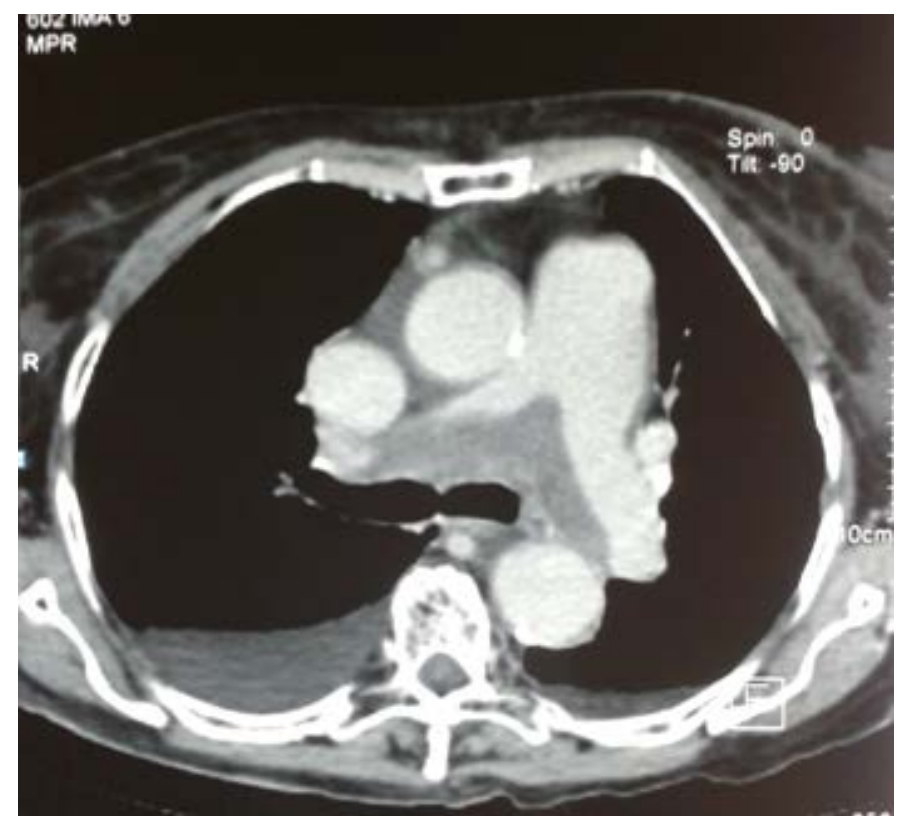

Figure 4. Augmentation of the true lumen diameter in the Left pulmonary artery trunk after 6 months of conservative treatment. 
transthoracic echocardiography is very contributive. Thoracic MRI provides more accurate anatomical approach, but it is useful in case of aneurysm of the pulmonary artery completely thrombosed, poorly visualized on CT [4].

Due to the low number of reported cases in the literature, the best treatment for patients with PAD has not yet been defined. Only a few patients have been treated surgically and several surgical approaches have been used [12]. The own evolutionary engineering pulmonary disease is discussed, favorable for some, pejorative for others, requiring the intervention after diagnosis [10]. Patients with a known history of pulmonary hypertension should be closely followed. The diameter of the dilated pulmonary artery should be monitored because it is directly related to the risk of rupture or dissection. In these patients, a pharmacologic treatment to reduce the pulmonary hypertension would help to reduce the risk of pulmonary artery dilatation. The outcome of patients with PAD treated surgically is related to the clinical condition when the patients come to surgery. For those patients who undergo successful surgical treatment of PAD, survival is high at short-term follow up [12]. Patients with PAD who are not deemed to be suitable for surgery are treated with medical therapy with diuretics and vasodilators. However, despite a few cases successfully treated by conservative management, the risk of rupture into the pericardium or other intrathoracic structures, leading to sudden death, should be kept in mind [13]. The justification or the constraint on the choice of medical treatment in our context is the absence of adequate surgical equipment to carry out such a complex surgical procedure. The patient still alive and clinically more improved. She is frequently followed up including clinical examination, echocardiography and CT scan.

\section{Conclusion}

The pulmonary artery dissection is a rare, not well known and life threatening event that should be suspected in any patient with chest pain, worsening dyspnea, or hemodynamic compromise with chronic pulmonary hypertension. It is typically diagnosed at post mortem autopsy, but with the advances in non invasive imaging techniques (CT; MRI), diagnostic research in patients during life is increasingly observed. In particular, successful treatment by conservative management in PAD patients can be obtained, but the risk of rupture leading to sudden death remains.

\section{References}

[1] Deb, S.J., Zehr, K.J. and Shields, R.C. (2005) Idiopathic Pulmonary Artery Aneurysm. The Annals of Thoracic Surgery, 80, 1500-1502. http://dx.doi.org/10.1016/j.athoracsur.2004.04.011

[2] Ben Ghorbel, I., Ibn Elhadj, Z., Khanfir, M. and Houman, M.H. (2004) Pulmonary Arterial Aneurysms in Behçet's Syndrome. Report of 4 Cases. Arch Mal Cour Vaiss, 97, 1195-1199.

[3] Ay, Y., Ay, N.K., Aydin, C., et al. (2013) A Rare Complication of Pre-Eisenmenger Patent Ductus Arteriosus: Pulmonary Artery Dissection. International Journal of Surgery Case Reports, 4, 483-485. http://dx.doi.org/10.1016/j.ijscr.2013.02.011

[4] Senbaklavaci, O., Kaneko, Y., Bartunek, A., et al. (2001) Rupture and Dissection in Pulmonary Artery Aneurysms: Incidence, Cause, and Treatment-Review and Case Report. The Journal of Thoracic and Cardiovascular Surgery, 121, 1006-1008. http://dx.doi.org/10.1067/mtc.2001.112634

[5] Yamamoto, M.E., Jones, J.W. and McManus, B.M. (1988) Fatal Dissection of the Pulmonary Trunk: An Obscure Consequence of Chronic Pulmonary Hypertension. American Journal of Cardiovascular Pathology, 1, 353-359.

[6] Inayama, Y., Nakatani, Y. and Kitamura, H. (2001) Pulmonary Artery Dissection in Patients without Underlying Pulmonary Hypertension. Histopathology, 38, 435-442. http://dx.doi.org/10.1046/j.1365-2559.2001.01129.x

[7] Lopez-Candales, A., Kleiger, R.E., Aleman-Gomez, J., et al. (1995) Pulmonary Artery Aneurysm: Review and Case Report. Clinical Cardiology, 18, 738-740. http://dx.doi.org/10.1002/clc.4960181211

[8] Rosenson, R.S. and Sutton, M.S.J. (1986) Dissecting Aneurysm of the Pulmonary Artery Trunk in Mitral Stenosis. American Journal of Cardiology, 58, 1140-1141. http://dx.doi.org/10.1016/0002-9149(86)90142-6

[9] Baptista, R., Castro, G., da Silva, A.M., et al. (2012) Pulmonary Dissection during Diagnostic Pulmonary Angiography. Revista Portuguesa de Cardiologia, 31, 465. http://dx.doi.org/10.1016/j.repc.2012.04.004

[10] Steurer, J., Jenni, R., Medici, T.C., et al. (1990) Dissecting Aneurysm of the Pulmonary Artery with Pulmonary Hypertension. American Review of Respiratory Disease, 142, 1219-1221. http://dx.doi.org/10.1164/ajrccm/142.5.1219

[11] Bhatia, V., Sharma, S., Panda, P., et al. (2014) Role of Multi-Detector Computed Tomography (mdet) in Diagnosis of Pulmonary Artery Dissection: A Rare but Fatal Entity. Annals Academy of Medicine Singapore, 43, 64-65. 
[12] Tuncer, A., Tuncer, E.Y., Tas, S.G., et al. (2011) Repair of Pulmonary Artery Aneurysms. Journal of Cardiac Surgery, 26, 501-505. http://dx.doi.org/10.1111/j.1540-8191.2011.01302.x

[13] Rousou, A.J., Haddadin, A., Badescu, G., et al. (2010) Surgical Repair of Pulmonary Artery Dissection. European Journal Cardio-Thoracic Surgery, 38, 805. http://dx.doi.org/10.1016/j.ejcts.2010.03.064 